\title{
HIF-1 $\alpha$ activation under glucose deprivation plays a central role in the acquisition of anti-apoptosis in human colon cancer cells
}

\author{
ARATA NISHIMOTO $^{1}$, NARUJI KUGIMIYA ${ }^{1}$, TOHRU HOSOYAMA ${ }^{1}$, \\ TADAHIKO ENOKI ${ }^{1}$, TAO-SHENG LI $^{2}$ and KIMIKAZU HAMANO ${ }^{1}$ \\ ${ }^{1}$ Department of Surgery and Clinical Science, Yamaguchi University Graduate School of Medicine, Ube, Yamaguchi 755-8505; \\ ${ }^{2}$ Department of Stem Cell Biology, Nagasaki University Graduate School of Biomedical Sciences, Nagasaki 852-8523, Japan
}

Received December 4, 2013; Accepted January 29, 2014

DOI: $10.3892 /$ ijo.2014.2367

\begin{abstract}
A poor vascular network development in a tumor mass leads to poor oxygen and nutrient supply. To adapt to a hypoxic microenvironment, it is well-known that cancer cells activate the transcription factor hypoxia-inducible factor- $1 \alpha$ (HIF-1 $\alpha$ ). HIF-1 $\alpha$ plays a central role in hypoxia-induced metabolic switching, anti-apoptosis, angiogenesis and drug resistance. Glucose deprivation, another major stressful microenvironment, protects cancer cells from drug-induced apoptosis. However, the molecular mechanisms by which cancer cells adapt to poor nutrient conditions remain poorly understood. In this study, we focused on HIF-1 $\alpha$, signal transducer and activator of transcription 3 (STAT3) and transcription factor 4 (TCF4), which are involved in cell survival, anti-apoptosis and drug resistance. We examined their activities and the relationships among these transcription factors under glucose deprivation. Our results showed that glucose deprivation increased HIF-1 $\alpha$, STAT3 and TCF4 DNA-binding activity, as well as the expression levels of their target genes $O C T 4, B C L-2$ and VEGF. HIF-1 $\alpha$ knockdown significantly increased poly(ADP-ribose) polymerase 1 (PARP-1) cleavage at higher levels than STAT3 knockdown under glucose deprivation. Furthermore, HIF-1 $\alpha$ knockdown led to a significant decrease in the expression levels of both STAT3 and TCF4, although STAT3 knockdown decreased only HIF-1 $\alpha$ expression level. Our data indicated that activation of the HIF-1 $\alpha$ signaling pathway under glucose deprivation leads to the acquisition of anti-apoptotic properties in human colon cancer cells, and targeting the HIF-1 $\alpha$ signaling pathway may provide an effective avenue for treating cancers resistant to conventional therapy.
\end{abstract}

Correspondence to: Dr Arata Nishimoto, Department of Surgery and Clinical Science, Yamaguchi University Graduate School of Medicine, 1-1-1 Minami-Kogushi, Ube, Yamaguchi 755-8505, Japan E-mail: anishimo@yamaguchi-u.ac.jp

Key words: glucose deprivation, anti-apoptosis, HIF-1 $\alpha$, STAT3, TCF4

\section{Introduction}

It is widely acknowledged that most cancer cells are exposed to hypoxia. Distinct from normal cells, most cancer cells predominantly produce energy by glycolysis, which is irrespective of oxygen availability, rather than oxidative phosphorylation via the tricarboxylic acid cycle (Warburg effect) (1). This is a reason why cancer cells can adapt to hypoxic condition and survive under hypoxia. Furthermore, these findings indicate that hypoxia in tumor is caused not by the excessive oxygen consumption of cancer cells, but rather the inadequate blood supply resulting from structurally and functionally defective angiogenesis. Consequently, poor vascularization in solid tumors leads to a poor supply of nutrients besides oxygen. Indeed, recent study has revealed that glucose concentration in tumor is lower than that in normal tissue (2).

When cancer cells are exposed to low oxygen condition, hypoxia-inducible factor- $1 \alpha(\mathrm{HIF}-1 \alpha)$ is activated, and its activation leads to cell survival under hypoxic condition through inducing transcriptions of a series of genes for anaerobic glycolysis, angiogenesis, and anti-apoptosis (3). Hypoxia can also enhance the activity of other transcription factors, including c-Jun, nuclear factor- $\kappa \mathrm{B}(\mathrm{NF} \kappa \mathrm{B}), \mathrm{SP} 1$, activator protein 1 (AP-1), signal transducer and activator of transcription 3 (STAT3), and STAT5 (4-6), which are known to be critical for cell survival and anti-apoptosis. On the other hand, glucose deprivation, which is another stressful microenvironment, enhances expression of the multidrug resistance-1 (MDR1) gene through c-Jun activation (7) and protects cancer cells from cisplatin-induced apoptosis by enhancing expression of asparagine synthetase (8). However, the network of transcription factors activated by glucose deprivation has not been fully elucidated compared with that of hypoxia.

In this study, we investigated how glucose deprivation affects the HIF-1 $\alpha$, STAT3, and transcription factor 4 (TCF4) signaling pathways involved in cell survival, anti-apoptosis, and drug resistance (9-11). We also further examined their roles in glucose deprivation-induced anti-apoptosis and the relationships among these transcription factors.

\section{Materials and methods}

Cell line and antibodies. The human colon cancer cell line COLO-320 was purchased from RIKEN Bioresource Center 
(Ibaraki, Japan) and maintained in RPMI-1640 medium containing glucose and supplemented with $10 \%$ fetal calf serum (FCS) at $37^{\circ} \mathrm{C}$ in a humidified atmosphere containing $5 \% \mathrm{CO}_{2}$. Mouse monoclonal anti-PARP-1 (F-2) antibody, mouse monoclonal anti-tubulin (B-5-1-2) antibody, mouse monoclonal anti-TCF-4 (YY-71) antibody, and rabbit polyclonal anti-STAT3 (C-20) antibody were purchased from Santa Cruz Biotechnology, Inc. (Santa Cruz, CA, USA). Mouse monoclonal anti-HIF1- $\alpha$ [H1 $\alpha 67]-C h I P$ Grade (ab1) antibody was purchased from Abcam (Cambridge, UK). Goat polyclonal anti-mouse $\mathrm{IgG}$ and anti-rabbit IgG antibody conjugated with horseradish peroxidase (HRP) were purchased from Dako (Carpinteria, CA, USA).

Cell culture under glucose deprivation. For glucose deprivation, COLO-320 cells were incubated for $48 \mathrm{~h}$ in RPMI-1640 medium containing $2 \mathrm{~g} / 1$ glucose (control condition) or glucose-free RPMI-1640 medium, which was supplemented with $5 \% \mathrm{FCS}$ at $37^{\circ} \mathrm{C}$ in a humidified atmosphere containing $5 \% \mathrm{CO}_{2}$.

Cell viability under glucose depletion. To investigate cell viability, cells were seeded in 6 -well plates $\left(3 \times 10^{6}\right.$ cells/well) under control condition or glucose deprivation. Following incubation for $48 \mathrm{~h}$, the cells were harvested using a cell scraper and suspended in phosphate-buffered saline (PBS). The cell suspension was mixed with an equal amount of trypan blue solution and the number of living cells was counted.

Western blotting. Cells were lysed with 1\% NP-40 lysis buffer [50 mM Tris-HCl (pH 7.4), 150 mM NaCl, 1\% (v/v) Nonidet $\mathrm{P}-40$ and $1 \mathrm{X}$ protease inhibitor] and incubated on ice for $30 \mathrm{~min}$. After centrifugation, cell lysates containing $20 \mu \mathrm{g}$ protein were dissolved in sample buffer solution with reducing reagent (Nacalai Tesque, Kyoto, Japan) and incubated at room temperature for $20 \mathrm{~min}$. Cell lysates dissolved in sample buffer were separated using SDS-PAGE and transferred to a polyvinylidene fluoride (PVDF) membrane. After blocking with Tris-buffered saline containing Tween-20 (TBST) containing $0.3 \%$ milk, the membrane was immunoblotted with the appropriate primary antibody, followed by goat anti-mouse immunoglobulins (Igs) or anti-rabbit Igs conjugated with horseradish peroxidase (HRP). After washing, immune complexes were detected using Amersham ECL Prime Western Blotting Detection reagent (GE Healthcare, Little Chalfont, UK).

Preparation for nuclear extracts and cytoplasmic extracts. Nuclear extracts and cytoplasmic extracts were prepared from COLO-320 cells cultured under control condition or glucose deprivation using the Nuclear Complex Co-IP kit (Active Motif, Carlsbad, CA, USA) according to the manufacturer's protocol.

Gel shift assay. Nuclear extracts were prepared from COLO-320 cells cultured under control condition or glucose deprivation. A gel shift assay was conducted using a doublestranded, biotin-labeled oligonucleotide probe containing the consensus binding site for STAT3 (sense strand, 5'-GATCCTT CTGGGAATTCCTAGATC-3'), HIF-1 $\alpha$ (sense strand, 5'-TCT GTACGTGACCACACTCACCTC-3') or TCF4 (sense strand,
5'-GGCTTTGAAGTATGA-3') using the Gelshift Chemiluminescent EMSA kit(Active Motif) according to the manufacturer's protocol. Protein-DNA complexes were resolved on a nondenaturing polyacrylamide gel, transferred to a positively charged nylon membrane, and cross-linked to a membrane using the UV-light cross-linker. After blocking, the membrane was incubated with blocking buffer containing streptavidin conjugated to HRP. After washing, protein-DNA complexes were detected using a chemiluminescent substrate (Active Motif).

Reverse transcription-polymerase chain reaction. Total RNAs were purified from COLO-320 cells cultured under control condition or glucose depletion using the RNeasy mini kit (Qiagen, Hilden, Germany) according to the manufacturer's protocol. Synthesis of cDNA and subsequent PCR were conducted using PrimeScript One Step RT-PCR Kit Ver. 2 (Takara, Shiga, Japan) according to the manufacturer's protocol. The sequences of primers used in this study were as follows: OCT4, 5'-ACACCTGGCTTCGGATTTCG-3' and 5'-GGCG ATGTGGCTGATCTGCT-3'; NANOG, 5'-AACATGAGTGT GGATCCAG-3' and 5'-TCACTCATCTTCACACGTCTTC AGGTTG-3'; $B C L-2$, 5'-AGATGTCCAGGCAGCTGCACCT GAC-3' and 5'-ATAGGCACCCAGGGTGATGCAAGCT-3'; $V E G F$, 5'-TCGGGCCTCCGAAACCATGA-3' and 5'-CCT GGTGAGAGATCTGGTTC-3'; GAPDH, 5'-GGAAGGTG AAGGTCGGAGTC-3' and 5'-GAAGATGGTGATGGGAT TTC-3'. Reaction conditions for each primer set were $50^{\circ} \mathrm{C}$ for $30 \mathrm{~min}$ and $94^{\circ} \mathrm{C}$ for 2 min followed by 20 cycles $(G A P D H)$, 24 cycles $(O C T 4, N A N O G), 25$ cycles $(V E G F)$ or 29 cycles (BCL-2) of the following reaction: denaturing step at $94^{\circ} \mathrm{C}$ for $30 \mathrm{sec}$; annealing at $55^{\circ} \mathrm{C}(N A N O G, G A P D H), 58^{\circ} \mathrm{C}(V E G F)$, $65^{\circ} \mathrm{C}(O C T 4)$ or $70^{\circ} \mathrm{C}(B C L-2)$ for $30 \mathrm{sec}$; and extension at $72^{\circ} \mathrm{C}$ for $30 \mathrm{sec}$. PCR products were analyzed on a $1 \%$ agarose gel containing ethidium bromide and detected using a UV transilluminator.

Clinical colorectal cancer specimens. Patients with colorectal cancer who underwent surgical treatment at Yamaguchi University and affiliated hospitals between April, 2012 and September, 2012 were enrolled in this study. Resected tumors specimens were immediately taken from resected colons and kept at $-80^{\circ} \mathrm{C}$ until RNA extraction. These samples were used in accordance with institutional guidelines and the Declaration of Helsinki after obtaining informed consent from all patients.

Knockdown of target gene expression by siRNA transfection. For siRNA transfection, scrambled (control), STAT3 or HIF-1 $\alpha$ siRNA (Thermo Scientific Dharmacon, Lafayette, CO, USA) was mixed with Lipofectamine RNAiMAX Reagent (Life Technologies, Carlsbad, CA, USA) in serum-free RPMI-1640 medium, and then the siRNA solution was incubated for $20 \mathrm{~min}$ at room temperature to form the siRNA-cationic lipid complex. Trypsinized COLO-320 cells were suspended with RPMI-1640 medium containing 10\% FCS and mixed with the siRNA solution (reverse transfection). Following incubation with siRNA at concentrations of $100 \mathrm{nM}$ for 2 days, siRNAtransfected COLO-320 cells were spread and incubated for 3 more days. After that, the cells were harvested using a cell scraper and resuspended in PBS. Following the centrifugation 
A

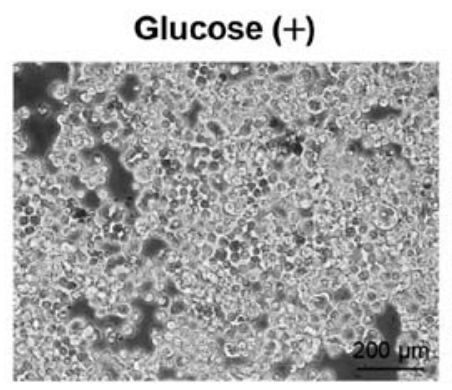

Glucose (-)

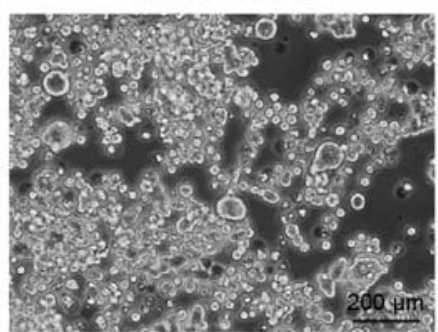

C

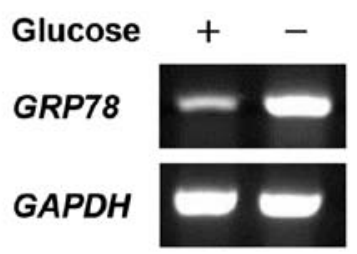

B

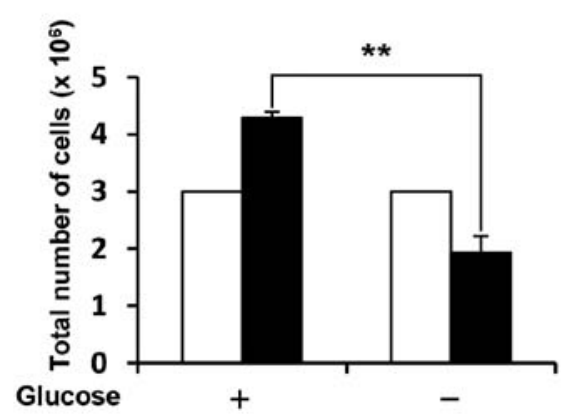

D

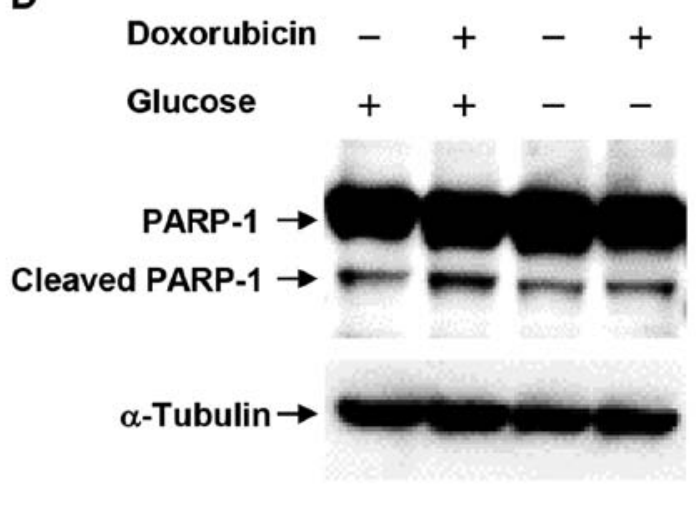

Figure 1. Effect of glucose deprivation on cellular morphology, cell number and drug resistance of human colon cancer cell line COLO-320 cells. COLO-320 cells were incubated under control condition or glucose deprivation for $48 \mathrm{~h}$. (A) Cellular morphology of COLO-320 cells incubated under control condition or glucose deprivation. Representative phase contrast images were taken at x 200 magnification. Scale bars indicate $200 \mu \mathrm{m}$. (B) Change in living cell number of COLO-320 cells incubated under control condition or glucose deprivation. Empty bar or filled bar indicates the cell number at the time of seeding or after $48 \mathrm{~h}$, respectively. Each bar represents the mean $\pm \mathrm{SD}$ of three independent experiments. ${ }^{* *} \mathrm{p}<0.01$, significantly different (n=3). (C) Total RNA was purified from COLO-320 cells incubated under control condition or glucose deprivation for $48 \mathrm{~h}$, and the expressions of GRP78 and GAPDH were detected using RT-PCR. (D) After cell culture under control condition or glucose deprivation for $24 \mathrm{~h}$, COLO-320 cells were treated with or without doxorubicin (Sigma, St. Louis, MO, USA) at concentrations of $10 \mu \mathrm{M}$ for $24 \mathrm{~h}$ under control condition or glucose deprivation. PARP-1 and cleaved PARP-1 were detected in total cell lysates using western blotting with anti-PARP-1 antibody. $\alpha$-tubulin was used to assess the total amount of proteins loaded on the gel.

at $1200 \mathrm{rpm}$ for $3 \mathrm{~min}$, the supernatants were removed and the siRNA-transfected cell pellets were used to prepare total cell lysates, nuclear extracts or cytoplasmic extracts for western blotting.

Statistical analysis. Data are expressed as means \pm standard deviation (SD). Statistical comparisons between groups were conducted using Student's t-test. Values of $\mathrm{p}<0.05$ or $\mathrm{p}<0.01$ were considered statistically significant.

\section{Results}

Human colon cancer cells adapted to glucose deprivation acquire resistance to doxorubicin-induced apoptosis. Following cell culture under glucose deprivation for $48 \mathrm{~h}$, the cell number of the survived COLO-320 cells was approximately half compared with the control (Fig. 1A and B). To confirm that COLO-320 cells adapt to glucose deprivation, we examined the mRNA expression level of glucose-regulated protein 78 (GRP78), which is well-known to be induced by stressful microenvironments such as glucose deprivation and hypoxia (12). As shown in Fig. 1C, the expression level of GRP78 was increased under glucose deprivation. These results indicate that the survived COLO-320 cells can adapt to glucose deprivation. Next, to investigate whether the survived COLO-320 cells under glucose deprivation could acquire drug resistance, the cells were treated with $10 \mu \mathrm{M}$ doxorubicin for $24 \mathrm{~h}$, followed by examination of PARP-1 cleavage, which is an indicator for apoptosis. As shown in Fig. 1D, PARP-1 cleavage was induced by doxorubicin treatment in cells cultured under control condition, and doxorubicin-induced PARP-1 cleavage was lower in cells cultured under glucose deprivation than that in the control cells. This result suggests that glucose deprivation confers resistance to doxorubicin-induced apoptosis.

Glucose deprivation increases DNA-binding activity of HIF-1 $\alpha$, STAT3, and TCF4 as well as the expression levels of their target genes. Gel shift assays using nuclear extracts revealed that HIF-1 $\alpha$, STAT3 and TCF4 DNA-binding activity in glucose-depleted COLO-320 cells were higher than those in the control cells (Fig. 2A-C). To further investigate the effect of glucose deprivation on the expression levels of HIF-1 $\alpha$, STAT3 and TCF4 target genes, we prepared total RNA from cells cultured under control condition or glucose deprivation, followed by RT-PCR. We selected HIF-1 $\alpha$, STAT3 or TCF4 target genes, which were previously demonstrated to be involved in anti-apoptosis and drug resistance, including OCT4 as HIF-1 $\alpha$ target gene (13), BCL-2 as STAT3 target 
A

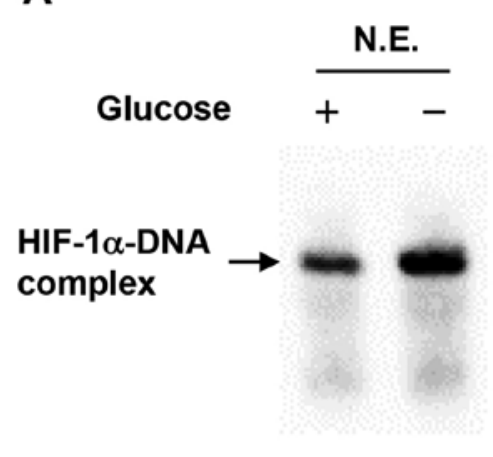

B

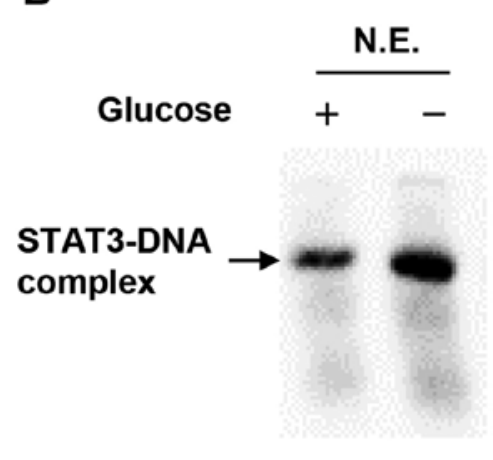

C

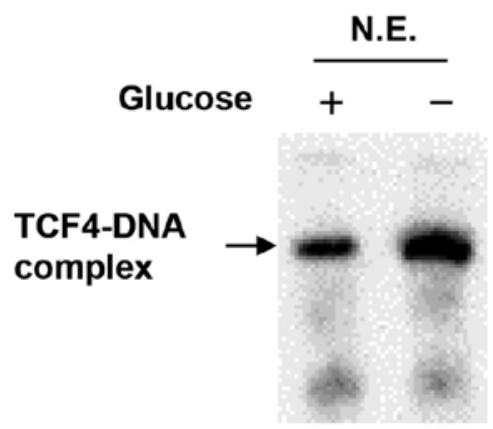

D

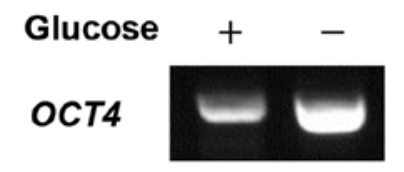

$B C L-2$
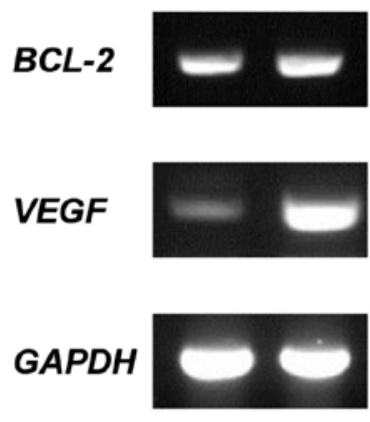

H

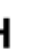

E

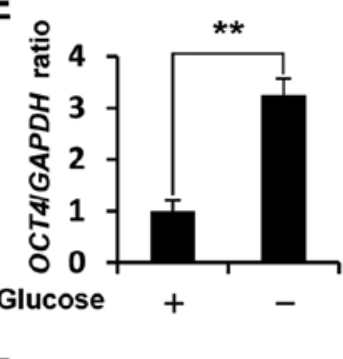

$\mathbf{F}$

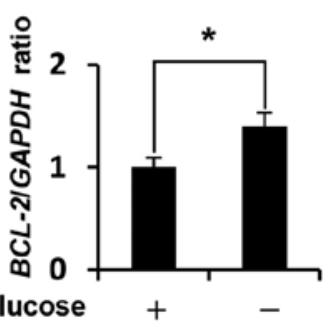

Glucose

G

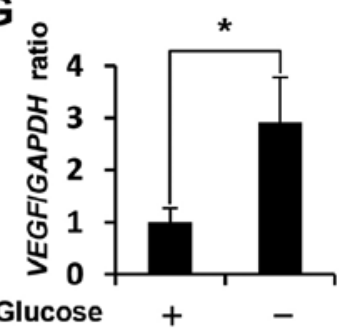

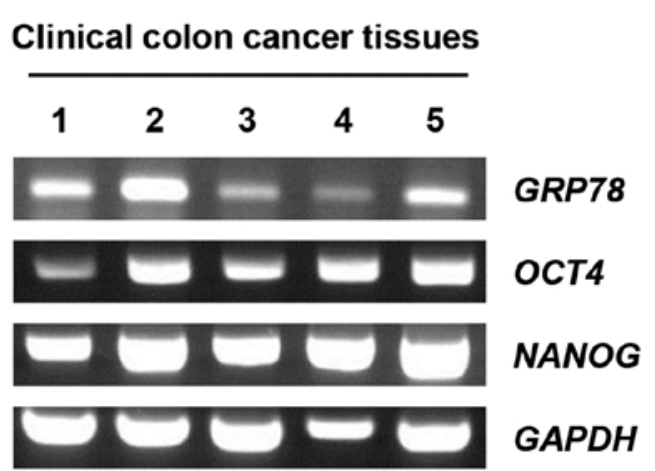

Figure 2. Effect of glucose deprivation on HIF-1 $\alpha$, STAT3, TCF4 DNA-binding activity, and the expression levels of their target genes. (A-C) Nuclear extracts were prepared from COLO-320 cells incubated under control condition or glucose deprivation for $48 \mathrm{~h}$. HIF-1 $\alpha$, STAT3 or TCF4 bound to biotin-labeled target DNA was detected using a gel shift assay. N.E., nuclear extracts. (D) Total RNA was purified from COLO-320 cells incubated under control condition or glucose deprivation for $48 \mathrm{~h}$, and the expressions of $O C T 4, B C L-2, V E G F$, and $G A P D H$ were detected using RT-PCR. (E-G) The intensity of each band was quantified using Image $\mathrm{J}$ software. The ratio of $O C T 4, B C L-2$ or $V E G F$ to $G A P D H$ was normalized to the values in control cells. Each bar represents the mean $\pm \mathrm{SD}$ of three independent experiments. ${ }^{*} \mathrm{p}<0.05,{ }^{* * *} \mathrm{p}<0.01$, significantly different $(\mathrm{n}=3)$. $(\mathrm{H})$ Total RNA was purified from clinical colon cancer tissues, and the expressions of GRP78, OCT4, NANOG, and GAPDH were detected using RT-PCR.

gene (14), and VEGF as HIF-1 $\alpha$, STAT3, and TCF4 target gene (15-17). Our results showed that glucose deprivation significantly increased the expression levels of these target genes (Fig. 2D-G) at 1.4-3.25-fold higher compared with those in COLO-320 cells cultured under control condition. Furthermore, we examined the expression levels of GRP78, $O C T 4$ and NANOG which is well-known as OCT4 target gene (18) in clinical colon cancer tissues. GRP78 is induced in the tumor microenvironment such as hypoxia and glucose deprivation, therefore it may be possible to estimate the tumor microenvironment in which clinical colon cancer tissues have grown by means of GRP78 expression level. As shown in Fig. 2H, GRP78 expression level in some clinical colon cancer tissues were higher than that in others, and OCT4 and its target gene, $N A N O G$ expression levels tended to correlate with GRP78 expression level.

HIF-1a knockdown significantly increases apoptosis in glucose-depleted COLO-320 cells. To investigate whether acquisition of anti-apoptotic property in glucose-depleted COLO-320 cells was due to activation of HIF-1 $\alpha$ or STAT3, we performed siRNA transfection studies. Glucose-depleted 
A

B

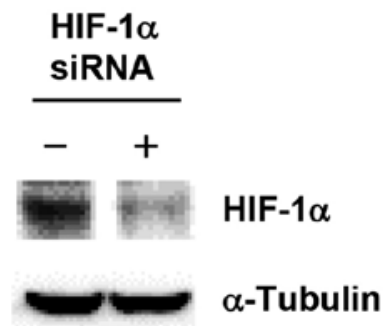
$-+$

STAT3
C Scrambled siRNA + - - + - STAT3 SIRNA $-+-\quad+\quad+$ HIF-1 $\alpha$ SiRNA

$\alpha$-Tubulin

PARP-1

Cleaved PARP-1
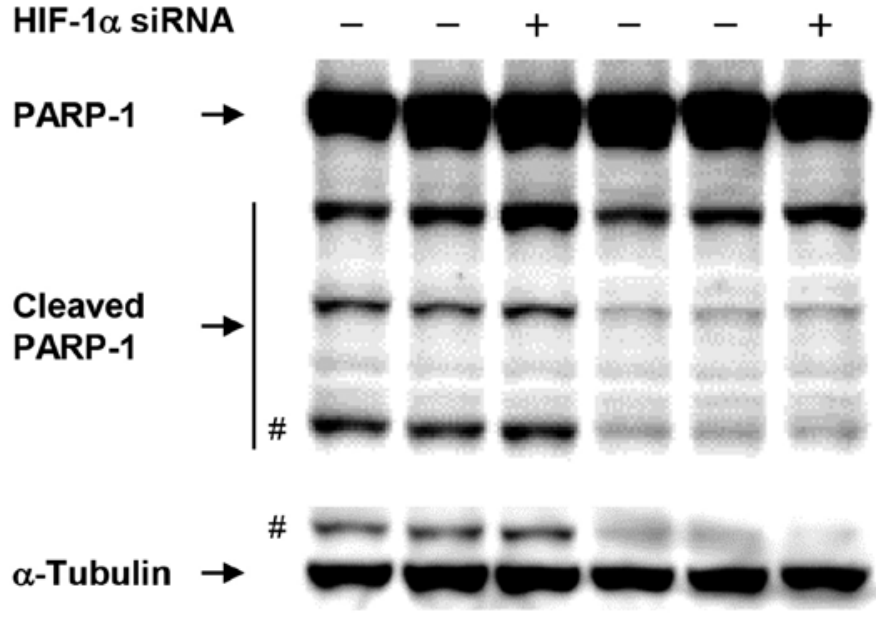

D

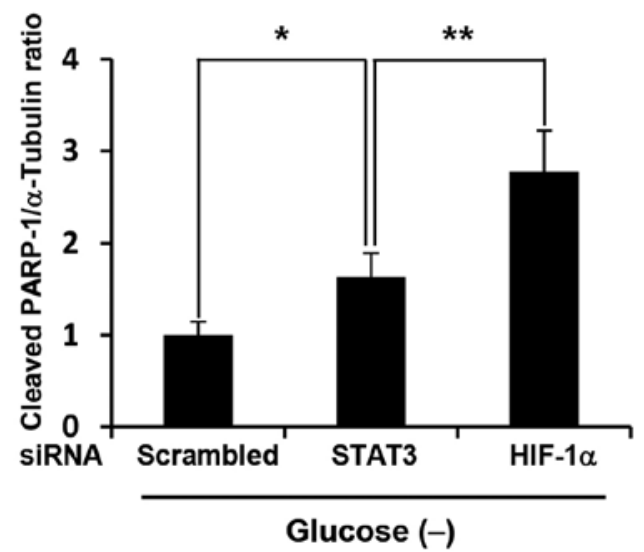

Figure 3. Comparison of PARP-1 cleavage between STAT3- and HIF-1 $\alpha$-silenced COLO-320 cells incubated under glucose deprivation. COLO-320 cells transfected with scrambled siRNA, STAT3 siRNA or HIF-1 $\alpha$ siRNA were incubated under glucose deprivation for $48 \mathrm{~h}$, and total cell lysates were prepared. (A and B) STAT3 and HIF-1 $\alpha$ were detected in total cell lysates using western blotting with anti-STAT3 and HIF-1 $\alpha$ antibody, respectively. $\alpha$-tubulin was used to assess the total amount of proteins loaded on the gel. (C) COLO-320 cells transfected with scrambled siRNA, STAT3 siRNA or HIF-1 $\alpha$ siRNA were incubated under control condition or glucose deprivation for $48 \mathrm{~h}$ and total cell lysates were prepared. PARP-1 and cleaved PARP-1 were detected in total cell lysates using western blotting with anti-PARP-1 antibody. $\alpha$-tubulin was used to assess the total amount of proteins loaded on the gel. Hash mark (\#) in the upper and the lower panels indicates the same band of cleaved PARP-1. (D) The intensity of each band was quantified using Image J software. The ratio of cleaved PARP-1 to $\alpha$-tubulin under glucose deprivation was normalized to the values in control cells. Each bar represents the mean \pm SD of three independent experiments. ${ }^{*} \mathrm{p}<0.05,{ }^{* *} \mathrm{p}<0.01$, significantly different $(\mathrm{n}=3)$.

COLO-320 cells transfected with HIF-1 $\alpha$ or STAT3 siRNA showed a marked decrease in HIF-1 $\alpha$ or STAT3 expression level, respectively (Fig. 3A and B). As shown in Fig. 3C, HIF-1 $\alpha$ knockdown induced PARP-1 cleavage at the highest levels under both control condition and glucose deprivation, and the amount of cleaved PARP-1 under glucose deprivation was decreased compared with that under control condition, which indicates that anti-apoptotic property is increased under glucose deprivation. Moreover, HIF-1 $\alpha$ knockdown significantly increased PARP-1 cleavage compared with STAT3 knockdown under glucose deprivation (Fig. 3D). These results suggest that HIF-1 $\alpha$ plays an important role in the acquisition of anti-apoptotic property induced by glucose deprivation.

Cross-talk among HIF-1 $\alpha$, STAT3, and TCF4 in glucosedepleted COLO-320 cells. We further investigated how knockdown of HIF-1 $\alpha$ or STAT3 affected the expression levels of two other transcription factors. HIF-1 $\alpha$ - or STAT3-silenced COLO-320 cells were cultured under glucose deprivation, followed by western blotting using nuclear extracts and cytoplasmic extracts. Our results showed that HIF-1 $\alpha$ knockdown significantly decreased the expression levels of STAT3 and 

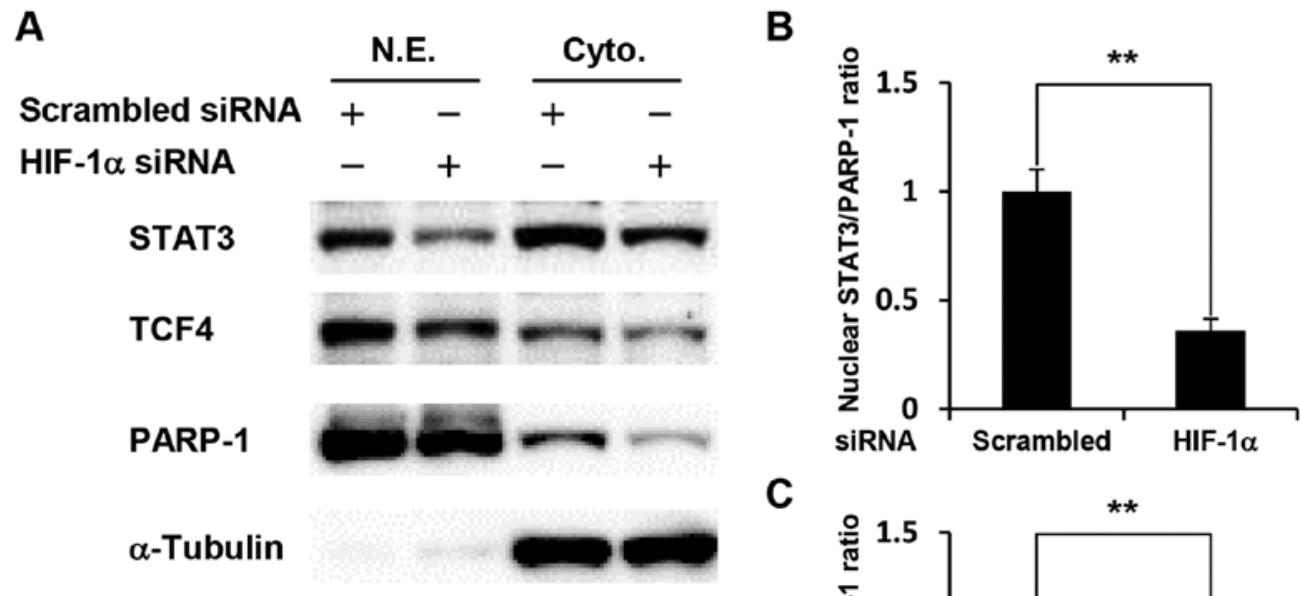

C
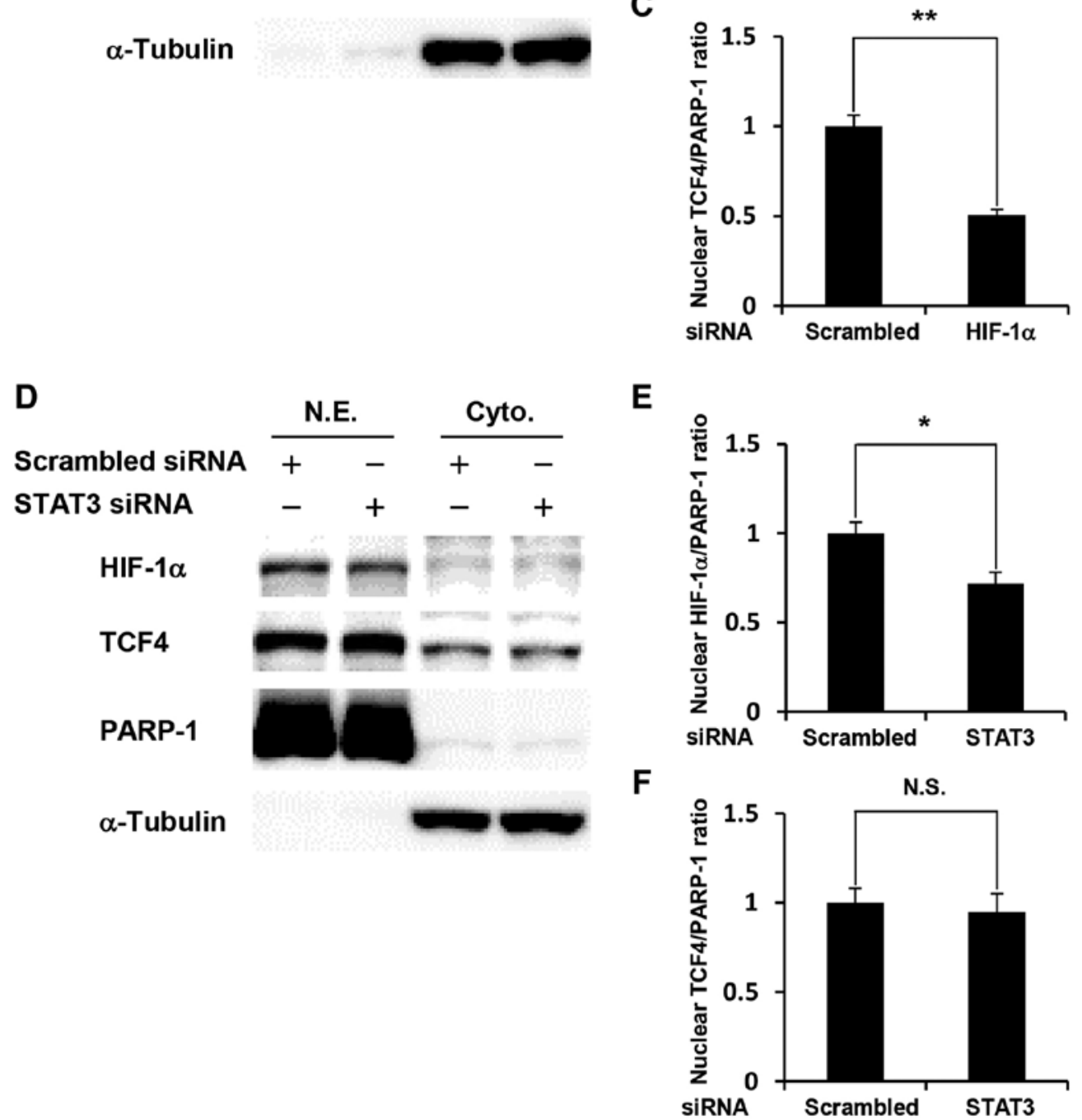

Figure 4. Cross-talk among HIF-1 $\alpha$, STAT3, and TCF4 in glucose-depleted COLO-320 cells. COLO-320 cells transfected with scrambled siRNA, STAT3 siRNA or HIF-1 $\alpha$ siRNA were incubated under glucose deprivation for $48 \mathrm{~h}$ and then harvested for preparation of nuclear and cytoplasmic extracts. (A and D) STAT3, TCF4 or HIF-1 $\alpha$ expression was detected in nuclear and cytoplasmic extracts using western blotting. PARP-1 or $\alpha$-tubulin was used to assess the total amount of nuclear or cytoplasmic extracts loaded on the gel, respectively. N.E., nuclear extracts. Cyto., cytoplasmic extracts. (B, C, E, and F) The intensity of each band was quantified using Image J software. The ratio of nuclear STAT3, HIF-1 $\alpha$ or TCF4 to PARP-1 was normalized to the values in control cells. Each bar represents the mean $\pm \mathrm{SD}$ of three independent experiments. ${ }^{*} \mathrm{p}<0.05,{ }^{* *} \mathrm{p}<0.01$, significantly different $(\mathrm{n}=3)$. N.S., no significant difference ( $\mathrm{n}=3$ ).

TCF4 in the nucleus (Fig. 4A-C). In contrast, STAT3 knockdown significantly decreased the expression level of HIF-1 $\alpha$ in the nucleus, but did not affect TCF4 expression (Fig. 4D-F). These results indicate that cross-talk among HIF-1 $\alpha$, STAT3, and TCF4 is involved in glucose deprivation-induced antiapoptosis.

\section{Discussion}

Therapeutic resistance remains a major obstacle in cancer therapy. To elucidate the mechanism by which colon cancer cells acquire drug resistance, we focused on the mechanism of the adaptation of colon cancer cells to glucose deprivation, 
which leads to the acquisition of resistance to drug-induced apoptosis.

Consistent with a previous study (19), 50\% of COLO-320 cells survived following glucose deprivation for $48 \mathrm{~h}$ and the survived COLO-320 cells under glucose deprivation acquired resistance to doxorubicin-induced apoptosis. These results suggest that the adaptation of colon cancer cells to glucose deprivation leads to the acquisition of resistance to drug-induced apoptosis. To elucidate the mechanism by which glucose deprivation confers resistance to drug-induced apoptosis, we further investigated how glucose deprivation affects the HIF-1 $\alpha$, STAT3, and TCF4 signaling pathways. The reasons why we focused on these signaling pathways are as follows. The HIF-1 $\alpha$ signaling pathway is well-known to be activated in response to hypoxia, which is another major stress microenvironment. The STAT3 signaling pathway is one of the major signaling pathways involved in regulation of cell survival and anti-apoptosis, and the TCF4 signaling pathway is critical for the development of colon cancer through the interaction between TCF4 and $\beta$-catenin (20). Interestingly, glucose deprivation activated all the three signaling pathways by increasing their DNA-binding activities as well as increasing the expression levels of their target genes, OCT4, $B C L-2$ and $V E G F$. Previous studies have shown that increased expression of BCL-2 and VEGF contributes to drug resistance $(21,22)$. Notably, among the upregulated genes, OCT4 is known to be highly and specifically expressed in normal stem cells. OCT4 is abundantly expressed in pluripotent stem cells, including embryonic stem cells and iPS cells, and has been shown to be necessary for maintaining the 'stemness' and pluripotency of stem cells (23). Coexpression of OCT4 and $N A N O G$ induces cancer stem-like properties including the ability to form sphere-like shapes, tumorigenicity, and drug resistance in lung adenocarcinoma (24). As cancer stemlike cells are highly resistant to chemotherapy (25), glucose deprivation may enhance drug resistance of cancer cells by changing their phenotype into that of cancer stem-like cells. In agreement with these data, the expression levels of OCT4 and its target gene $N A N O G$ tended to correlate with that of GRP78 in clinical colon cancer tissues. The clinical samples in which $G R P 78$ was highly expressed seemed to grow under hypoxia and glucose deprivation. In such clinical samples with high GRP78 expression, OCT4 and NANOG expression levels tended to be higher than those in other samples.

We found that HIF-1 $\alpha$ knockdown significantly induced PARP-1 cleavage at higher level than STAT3 knockdown in glucose-depleted colon cancer cells. It has been reported that HIF-1 $\alpha$ regulates the transcription of several genes involved in glycolysis $(26,27)$. Furthermore, recent studies have revealed that HIF-1 $\alpha$-mediated autophagy in oxygen- or nutrient-starved cancer cells promotes tumor cell survival and protects cancer cells from drug-induced apoptosis (28). Therefore, HIF-1 $\alpha$ knockdown in glucose-depleted colon cancer cells may disrupt glucose metabolism and autophagy induction, leading to apoptosis. Furthermore, we attempted to understand cross-talk among HIF-1 $\alpha$, STAT3, and TCF4 which are activated under glucose deprivation. Previous studies have demonstrated that STAT3 regulates HIF-1 $\alpha$ expression $(29,30)$. We also found that STAT3 knockdown significantly decreased the expression level of HIF-1 $\alpha$, but not TCF4, in glucose-depleted COLO-320 cells. Notably, HIF-1 $\alpha$ knockdown also significantly decreased the expression levels of STAT3 and TCF4, indicating the role of HIF-1 $\alpha$ as an important regulator in both the STAT3 and TCF4 signaling pathways. These results may explain why HIF-1 $\alpha$ knockdown induced PARP-1 cleavage at higher levels than STAT3 knockdown.

In conclusion, our data clearly show that glucose deprivation activates multiple transcription factors including HIF-1 $\alpha$, STAT3 and TCF4 in colon cancer cells. Among these transcription factors, HIF-1 $\alpha$ plays a central role in the acquisition of anti-apoptotic property under glucose deprivation and targeting the HIF-1 $\alpha$ signaling pathway may provide an effective avenue for treating colon cancer cells resistant to conventional therapy. However, glucose-depleted COLO320 cells did not completely undergo cell death by HIF-1 $\alpha$ knockdown alone. Further studies are necessary to identify key molecules that enhance the effect of HIF-1 $\alpha$ knockdown on anti-apoptosis of colon cancer cells resistant to conventional therapy.

\section{Acknowledgements}

We thank Dr K. Ueki (Yamaguchi Saiseikai Shimonoseki General Hospital, Shimonoseki, Japan) for assistance in acquiring clinical colon cancer tissues. This study was supported by Grant-in-Aids for Young Scientific Research (B) (no. 24791425 to A. Nishimoto) from Japan Society for the Promotion of Science (JSPS) and by a grant from Takeda Science Foundation of Japan.

\section{References}

1. Warburg O: On the origin of cancer cells. Science 123: 309-314, 1956.

2. Hirayama A, Kami K, Sugimoto M, et al: Quantitative metabolome profiling of colon and stomach cancer microenvironment by capillary electrophoresis time-of-flight mass spectrometry. Cancer Res 69: 4918-4925, 2009.

3. Zhou J, Schmid T, Schnitzer S and Brüne B: Tumor hypoxia and cancer progression. Cancer Lett 237: 10-21, 2006.

4. Cummins EP and Taylor CT: Hypoxia-responsive transcription factors. Eur J Physiol 450: 363-371, 2005.

5. Selvendiran K, Bratasz A, Kuppusamy ML, et al: Hypoxia induces chemoresistance in ovarian cancer cells by activation of signal transducer and activator of transcription 3 . Int $\mathrm{J}$ Cancer 125: 2198-2204, 2009.

6. Oh M-K, Park H-J, Kim N-H, et al: Hypoxia-inducible factor-1 $\alpha$ enhances haptoglobin gene expression by improving binding of STAT3 to the promoter. J Biol Chem 286: 8857-8865, 2011.

7. Ledoux S, Yang R, Friedlander G and Laouari D: Glucose depletion enhances P-glycoprotein expression in hepatoma cells: role of endoplasmic reticulum stress response. Cancer Res 63: 7284-7290, 2003.

8. Cui H, Darmanin S, Natsuisaka M, et al: Enhanced expression of asparagine synthetase under glucose-deprived conditions protects pancreatic cancer cells from apoptosis induced by glucose deprivation and cisplatin. Cancer Res 67: 3345-3355, 2007.

9. Duan Z, Foster R, Bell DA, et al: Signal transducer and activator of transcription 3 pathway activation in drug-resistant ovarian cancer. Clin Cancer Res 12: 5055-5063, 2006.

10. Liu L, Ning X, Sun L, et al: Hypoxia-inducible factor- $1 \alpha$ contributes to hypoxia-induced chemoresistance in gastric cancer. Cancer Sci 99: 121-128, 2008.

11. Kendziorra E, Ahlborn K, Spitzner M, et al: Silencing of the Wnt transcription factor TCF4 sensitizes colorectal cancer cells to (chemo-) radiotherapy. Carcinogenesis 32: 1824-1831, 2011.

12. Lee AS: GRP78 induction in cancer: therapeutic and prognostic implications. Cancer Res 67: 3496-3499, 2007. 
13. Mathieu J, Zhang Z, Zhou W, et al: HIF induces human embryonic stem cell markers in cancer cells. Cancer Res 71: 4640-4652, 2011.

14. Real PJ, Sierra A, Juan A, et al: Resistance to chemotherapy via Stat3-dependent overexpression of Bcl-2 in metastatic breast cancer cells. Oncogene 21: 7611-7618, 2002.

15. Forsythe JA, Jiang BH, Iyer NV, et al: Activation of vascular endothelial growth factor gene transcription by hypoxiainducible factor 1. Mol Cell Biol 16: 4604-4613, 1996.

16. Niu G, Wright KL, Huang M, et al: Constitutive Stat3 activity up-regulates VEGF expression and tumor angiogenesis. Oncogene 21: 2000-2008, 2002.

17. Hwang I, Kim J and Jeing S: $\beta$-catenin and peroxisome proliferator-activated receptor- $\delta$ coordinate dynamic chromatin loops for the transcription of vascular endothelial growth factor A gene in colon cancer cells. J Biol Chem 287: 41364-41373, 2012.

18. Rodda DJ, Chew J-L, Lim L-H, et al: Transcriptional regulation of Nanog by OCT4 and SOX2. J Biol Chem 280: 24731-24737, 2005.

19. Izuishi K, Kato K, Ogura T, et al: Remarkable tolerance of tumor cells to nutrient deprivation: possible new biochemical target for cancer therapy. Cancer Res 60: 6201-6207, 2000.

20. Korinek V, Barker N, Morin PJ, et al: Constitutive transcriptional activation by a $\beta$-catenin-Tcf complex in $\mathrm{APC}^{-/-}$colon carcinoma. Science 275: 1784-1787, 1997.

21. Reed JC: Regulation of apoptosis by bcl-2 family proteins and its role in cancer and chemoresistance. Curr Opin Oncol 7: 541-546, 1995.
22. Samuel S, Fan F, Dang LH, et al: Intracrine vascular endothelial growth factor signaling in survival and chemoresistance of human colorectal cancer cells. Oncogene 30: 1205-1212, 2011.

23. Loh Y-H, Ng J-H and Ng H-H: Molecular framework underlying pluripotency. Cell Cycle 7: 885-891, 2008.

24. Chiou S, Wang M-L, Chou Y-T, et al: Coexpression of Oct4 and Nanog enhances malignancy in lung adenocarcinoma by inducing cancer stem cell-like properties and epithelial-mesenchymal transdifferentiation. Cancer Res 70: 10433-10444, 2010

25. Vaiopoulos AG,Kostakis ID, Koutsilieris Mand Papavassiliou AG: Concise review: colorectal cancer stem cells. Stem Cells 30: 363-371, 2012.

26. Kim J-W and Dang CV: Cancer's molecular sweet tooth and the Warburg effect. Cancer Res 66: 8927-8930, 2006.

27. Kaelin WG Jr and Thompson CB: Clues from cell metabolism. Nature 465: 562-564, 2010

28. Liu X-W, Su Y, Zhu H, et al: HIF-1 $\alpha$-dependent autophagy protects Hela cells from fenretinide (4-HPR)-induced apoptosis in hypoxia. Pharmacol Res 62: 416-425, 2010.

29. Jung JE, Lee H-G, Cho I-H, et al: STAT3 is a potential modulator of HIF-1-mediated VEGF expression in human renal carcinoma cells. FASEB J 19: 1296-1298, 2005.

30. Xu Q, Briggs J, Park S, et al: Targeting Stat3 blocks both HIF-1 and VEGF expression induced by multiple oncogenic growth signaling pathways. Oncogene 24: 5552-5560, 2005. 